\title{
A Prognostic View on the Application of Individualized Genomics in Parkinson's Disease
}

\author{
Owen A. Ross
}

Published online: 12 January 2013

(C) Springer Science + Business Media New York 2013

\begin{abstract}
Parkinson's disease is a common age-related progressive neurodegenerative disorder. Over the last 15 years, advances have been made in our understanding of the etiology of the disease, with the greatest insights perhaps coming from genetic studies. The identification of a number of genes that harbor pathogenic mutations causing Parkinson's disease have on the whole driven the development of disease model systems and nominated a number of therapeutic targets. As we move towards an era of personalized medicine, genetic determinants will become even more crucial for accurate diagnosis, and assessing prognosis and outcomes. The individual genomic profile and risk assessments will in the long-term determine clinical trial participation, treatment plans and therapeutic dosing. Herein we discuss the status of genetics in Parkinson's disease and how these factors may affect patient care in the future.
\end{abstract}

Keywords Parkinson's disease - Genetics - Diagnosis · Individualized medicine $\cdot$ Genomics

\section{Introduction}

Since the original description of Parkinson's disease (PD), and the landmark essay by Parkinson in 1817 surprisingly little has changed with regards to the clinical description of the disease [1]. Almost two centuries later, however, we have a much better understanding of the natural history and pathophysiology of the disease $[2,3]$. The characterization

O. A. Ross ( $\square)$

Department of Neuroscience, Mayo Clinic, 4500 San Pablo

Road, Jacksonville, FL 32224, USA

e-mail: ross.owen@mayo.edu of the Lewy body as the pathologic lesion observed in the brain of patients with PD and the development of dopamine replacement therapies (levodopa) was the stand out advancements up until the last few years of the twentieth century $[4,5]$. As we moved towards the millennium, we entered a new era of research on PD which has been driven by genetic discovery. In fact, over the last decade, this era of 'genomics' has directed both functional biology and in vitro/in vivo modeling of the disease and as we progress will be critical for diagnostic, prognostic and therapeutic outcomes.

The diagnosis of PD relies on the clinical presentation of more than one of the four cardinal clinical signs; bradykinesia, rest tremor, rigidity and postural instability [6-8]. In addition, a positive response to dopamine replacement therapy is required. These criteria will lead to either a probable or possible diagnosis with definite PD requiring the presence of Lewy body pathology in the surviving neurons of the substantia nigra (referred to using the neuropathological definition as brainstem Lewy body disease; LBD) [9]. Given the many overlapping features with other forms of parkinsonism (PD is the most common form of parkinsonism) such as progressive supranuclear palsy (PSP) and multiple system atrophy (MSA), the clinical diagnosis of PD is not exact [10]. In addition, of course, waiting for the neuropathologic confirmation does not benefit the patient. The application of genetic testing is one approach that although in its infancy may circumvent many of the diagnostic issues and provide the neurologist with a clear path to therapeutic intervention.

Genetic discrimination of those individuals at risk will be crucial if the degeneration that is observed at the endstage of the disease begins well before the predominant clinical presentation, as suggested for Alzheimer's disease (AD) $\left[11^{\bullet}\right]$. There are many clinical signs that may predate 
Table 1 Reported familial PARK loci

\begin{tabular}{|c|c|c|c|c|}
\hline Locus & Region & Gene & Age-at-onset & Inheritance \\
\hline PARK1/4 & $4 q 21$ & SNCA & & AD \\
\hline PARK2 & $6 q 26$ & PRKN & Early-onset & $\mathbf{A R}$ \\
\hline PARK3 & $2 \mathrm{p} 13$ & - & & $\mathrm{AD}$ \\
\hline PARK5 & $4 \mathrm{p} 13$ & UCH-L1 & & $\mathrm{AD}$ \\
\hline PARK6 & $1 p 36.12$ & PINK1 & Early-onset & $\mathbf{A R}$ \\
\hline PARK7 & $1 p 36.23$ & DJ-1 & Early-onset & $\mathbf{A R}$ \\
\hline PARK8 & $12 p 12$ & LRRK2 & & AD \\
\hline PARK9 & $1 p 36.13$ & ATP13A2 & Early-onset & $\mathbf{A R}$ \\
\hline PARK10 & $1 \mathrm{p} 32$ & - & & - \\
\hline PARK11 & $2 \mathrm{q} 37.1$ & - & & $\mathrm{AD}$ \\
\hline PARK12 & Xq21-q25 & - & & X-linked \\
\hline PARK13 & $2 \mathrm{p} 13.1$ & - & & $\mathrm{AD}$ \\
\hline PARK14 & $22 q 13.1$ & PLA2G6 & Early-onset & $\mathbf{A R}$ \\
\hline PARK15 & $22 q 12.3$ & FBXO7 & Early-onset & $\mathbf{A R}$ \\
\hline PARK16 & $1 \mathrm{q} 32$ & - & & - \\
\hline PARK17 & $16 q 11.2$ & VPS35 & & AD \\
\hline PARK18 & $3 \mathrm{q} 27.1$ & EIF4G1 & & $\mathrm{AD}$ \\
\hline
\end{tabular}

PARK loci is the traditional nomenclature used to specify a chromosomal region that was linked with a specific parkinsonism phenotype. This system, however, is becoming outdated as classical linkage studies will most likely be replaced with next-generation sequencing technologies, and to date genome-wide association regions have not been designated as PARK loci. Those genes highlighted in bold are those confirmed to be a cause of PD and early-onset parkinsonism

$A D$ autosomal dominant; $A R$ autosomal recessive

the movement disorder component of PD; in fact, upwards of $60 \%$ of dopaminergic neurons are lost prior to the presentation of the movement disorder phenotype [12]. These early signs can include anosmia, constipation and REM sleep behavior disorders, however, the diagnostic utility of these signs remains controversial as they are present in many other disorders and are not specific to PD [13]. Imaging provides an additional weapon in the arsenal of the neurologist, but unfortunately visualization of $\alpha$ synuclein aggregation is unavailable in vivo at present. The development of amyloid- $\beta$ plaque (Pittsburgh compound $\mathrm{B}$; $\mathrm{PiB}$ ) imaging in $\mathrm{AD}$ has provided insight into the early stages of neurodegenerative disease. Studies suggest that the initial pathology can be observed approximately 25 years before the clinical onset of the disease [11•]. If this holds true in PD, it may indicate that disease intervention may need to be performed decades before the movement disorder manifests and present a major obstacle in treatment. Genetic susceptibility profiles may provide one mechanism whereby at-risk individuals can be identified in the prodromal stage, monitored and treated.

To apply clinical genetics to a disease, one must first however resolve the variation within the genome that is determining the individual susceptibility to disease. In the context of PD, genetic factors were long thought to play a minimal role, if any, given the largely sporadic nature of the disease [14]. However, we have witnessed a paradigm shift in the field following the identification of the first pathogenic mutation causing an autosomal dominantly inherited form of $\mathrm{PD}$ in the gene encoding the $\alpha$-synuclein protein (SNCA; Table 1) [15]. Subsequently, the $\alpha$-synuclein protein was demonstrated to be the major protein component of the Lewy body which places $\alpha$-synuclein at the center of PD research [4]. Over the following years it became clear that families with SNCA point mutations are rare. The identification of genomic multiplication of the SNCA gene as a dose-dependent mechanism of disease postulated the theory that overexpression of the wild-type $\alpha$-synuclein protein is sufficient to cause both familial and sporadic forms of PD [16, 17]. Indeed, subsequent population-based genetic studies have shown that common variation at the SNCA locus is a risk for sporadic disease [18, 19]; although caveats remain in identification of the true toxic $\alpha$-synuclein species [20•]. Together, these findings have nominated SNCA knockdown approaches as a viable therapeutic target and multiplication families may provide an ideal patient group for initial clinical trials [21].

Following the identification of $S N C A$ as a cause of autosomal dominant $\mathrm{PD}$, three genes were identified to cause early-onset forms of autosomal recessive parkinsonism that clinically reflect PD (PARKIN, PINK1 and $D J-1)$ [22]. Given that only approximately ten to fifteen percent of patients with PD present under the age of 45 years, carriers of mutations within these genes remain few, however, clinical genetic testing is available to the 
neurologist and can be utilized in the diagnosis. An issue within the setting of PD has been the elucidation of common pathways involving the mutated genes; while many cellular pathways have been implicated, the evidence has been unconvincing [23]. Recently, a novel mechanism for disease in PARKIN and PINK1 mutation carriers has been proposed involving the identification and clearance of damaged mitochondria via mitophagy, a specific form of autophagy [24•, 25, 26]. Studies have shown that PINK1 (a mitochondrial kinase) recruits Parkin (an E3 ubiquitin ligase) to damaged mitochondria for ubiquitination and then targeted clearance [27]. Although the role of this pathway in the more frequent late-onset form of PD remains unclear, this type of functional readout may be crucial in determining pathogenicity of rare variants.

Perhaps the most clinically relevant gene associated with PD identified to date was found in 2004 [28, 29]. The identification of mutations within the leucine-rich repeat kinase 2 gene (LRRK2) was notable for its clinical and pathologic presentation, which is indistinguishable from typical late-onset sporadic PD [30], and the frequency of its most studied mutant the LRRK2 G2019S substitution [3133]. The LRRK2 G2019S mutant accounts for between 5 and $6 \%$ of familial and $1 \%$ of sporadic patients in the North American Caucasian population [34]. Of note, in the Ashkenazi Jewish population and the Berber Arab populations of North Africa, this figure can increase upwards of 15 and $40 \%$, respectively [35, 36]. The substitution is predicted to increase the kinase activity of the protein and thus provides rationale for drug development [37, 38]. As with $S N C A$ a number of subsequent studies have identified common population variants in the LRRK2 gene which affect the individual's risk of developing disease [39, 40]. Our study last year working with the Global Epidemiology Of PD consortium (GEO-PD) identified a number of novel risk factors and evidence to support the presence of a protective $L R R K 2$ haplotype [41•].

As has been observed for the majority of pathogenic familial mutations, age-at-onset widely varies, and as with any late-onset disorder the phenomenon of age-related reduced penetrance is a major clinical issue. Penetrance is modified by other genetic or disease-modifying agents (environmental, stochastic) and is a characteristic feature of PD, observed for both SNCA and LRRK2 mutation carriers $[42,43]$. Indeed, healthy septuagenarian and octogenarian carriers of SNCA duplications and LRRK2 G2019S have been reported $[44,45]$. This wide spread in age-at-onset can be anywhere from 35 to 95 years in the case of LRRK2 G2019S, and highlights the potential to modify the penetrance of the disease in carriers. These observations provide tangible hopes in the development of disease-modifying therapies if they can be delivered to the correct patients at the correct intervention time in early-stage disease.
The vast majority of patients with PD report no family history and manifest a disease which is sporadic in nature. However, the genetic studies on familial forms of disease have also borne fruit in the nomination of common variants influencing risk of sporadic disease. In fact, the most significant findings from population-based association studies nominate common variation in SNCA and microtubuleassociated protein tau (MAPT) in which familial mutations have been shown to cause frontotemporal dementia linked to chromosome 17 with tau pathology [46•]. Early candidate gene-association studies also highlighted variation in SNCA and MAPT [47-49]. For many patients, it may be the joint effects of many low-penetrant risk and protective factors that determine the individual risk of developing PD. For example, a patient with the SNCA risk variants may also harbor the LRRK2 protective haplotype, with the combined sum of the effect reflecting the overall risk. Our recent study examined the independent and joint effects of SNCA and MAPT risk variants with members of the GEO-PD consortium [50॰]. The results suggest the genetic risk alleles at these two loci are independent, with an individual harboring both at an increased risk in an additive manner. Identifying the common variants has been a major challenge to the field, with only recent large-scale studies helping to define the genetic architecture of PD.

Early genome-wide association (GWA) efforts were on the whole disappointing; in 2011, the first large metaanalytical approach in GWA studies of PD was published nominating eleven loci (including SNCA, LRRK2 and $M A P T$ ) as risk determinants (Table 2) [46•,51•]. For the majority of association peaks, no individual gene or functional variant/s could be defined; therefore, measures of population-based risk should be treated with caution. The clinical utility of such observations is yet to be realized and may be irrelevant without the resolution of the specific variants driving the signal. These regions are large, contain numerous genes, and given that they were identified in population-based series rather than families, it is difficult to select only a small number of patients and controls for sequencing, as functional variants are likely present in both groups but not in everyone. Without a better understanding of the genome-wide susceptibility factors, it will be impossible to exploit the advantages of clinical genetic screening or to inform the patient what a positive test predicts regarding the potential of disease, i.e., life-time risk, age-at-onset, clinical phenotype.

Genetic studies are also likely to inform therapeutic intervention strategies and dosage. Unfortunately, the current medicines are focused on symptomatic alleviation (e.g., levodopa, dopamine agonists), with no effect on disease progression. As PD is a late-onset disease for the majority of patients (average age-at-onset is approximately 65 years), a therapy which can delay the onset by 
Table 2 Reported population-based GWA loci

\begin{tabular}{|c|c|c|c|c|c|}
\hline Chromosome & Genomic position $^{\mathrm{a}}$ & NCBI rs\# & Gene $^{\mathrm{b}}$ & $\mathrm{OR}^{\mathrm{c}}$ & References \\
\hline $1 q 22$ & 153451576 & N370S & GBA (N370S) & 3.08 & {$\left[61^{\bullet}\right]$} \\
\hline $1 q 22$ & 154105678 & & SYT11 & 1.67 & {$\left[46^{\bullet}\right]$} \\
\hline $1 q 32$ & 204019288 & rs947211 & $\underline{\text { PARK16 }}$ & 1.23 & {$[57]$} \\
\hline $2 q 24$ & 168800188 & rs2390669 & STK39 & 1.28 & {$\left[46^{\bullet}\right]$} \\
\hline $3 q 26$ & 160992864 & rs34016896 & NMD3 & 1.14 & {$\left[60^{\bullet}\right]$} \\
\hline $3 q 27$ & 184303969 & rs11711441 & MCCC1/LAMP3 & 0.82 & {$\left[46^{\bullet}\right]$} \\
\hline $4 \mathrm{p} 16$ & 954359 & rs11248060 & DGKQ & 1.69 & {$[55]$} \\
\hline $4 \mathrm{p} 15$ & 15737937 & rs4538475 & BST1 & 1.25 & {$[57]$} \\
\hline $4 q 21$ & 77198986 & rs6812193 & STBD1 & 0.89 & {$\left[60^{\bullet}\right]$} \\
\hline $4 q 22$ & 90606597 & rs356229 & SNCA & 1.35 & {$[55]$} \\
\hline $6 \mathrm{p} 21$ & 32409530 & rs3129882 & HLA-DRA & 1.31 & {$[56]$} \\
\hline $7 \mathrm{p} 15$ & 23306020 & rs 156429 & GPNMB & 0.89 & {$\left[60^{\bullet}\right]$} \\
\hline $8 q 21$ & 89442157 & & MMP16 & 1.38 & {$\left[60^{\bullet}\right]$} \\
\hline $8 q 22$ & 16697091 & rs591323 & FGF20 & 0.88 & {$\left[60^{\bullet}\right]$} \\
\hline $10 \mathrm{p} 13$ & 15601549 & rs7077361 & ITGA8 & 0.88 & {$[51]$} \\
\hline $12 q 12$ & 38907075 & rs1491942 & LRRK2 & 1.19 & {$\left[46^{\bullet}\right]$} \\
\hline $12 \mathrm{q} 24$ & 106949987 & rs4964469 & & 1.27 & {$[58]$} \\
\hline $12 q 24$ & 121892551 & rs10847864 & CCDC62/HIP1R & 1.16 & {$\left[46^{\bullet}\right]$} \\
\hline $16 \mathrm{p} 11$ & 30982225 & rs4889603 & STX1B & 1.12 & {$\left[60^{\bullet}\right]$} \\
\hline $17 \mathrm{p} 11$ & 17655826 & rs11868035 & SREBF1/RAI1 & 0.85 & [59] \\
\hline $17 q 21$ & 43781747 & rs1724425 & MAPT (H1/H2) & 0.68 & [55] \\
\hline $18 \mathrm{q} 12$ & 40673380 & rs12456492 & RIT2 & 1.19 & {$[61 \bullet]$} \\
\hline $21 \mathrm{q} 21$ & 15836776 & rs2823357 & USP25 & 1.15 & [59] \\
\hline
\end{tabular}

The loci/genes nominated to associate with PD susceptibility from genome-wide association studies

a The loci position is based on the 2009 human reference sequence (GRCh37; hg19 build)

$\mathrm{b}$ The nominated gene represents the best candidate or the nearest to the single nucleotide polymorphism with the lowest $p$ value and may not actually be the gene that harbors the functional variant/s which are associating with disease susceptibility. Underlined is the terminology for the locus on Chromosome 1q32 (PARK16), the use of the designated PARK loci may be best applied to some of the nominated GWA study regions

c The primary reported odds ratio (OR) from the original GWA study reference (Ref). Genes in which pathogenic mutations have been identified in familial forms of parkinsonism are highlighted in bold

10-20 years may be a considerable benefit to many individuals. In addition, presently patients can develop 'wearing off' with L-dopa therapy which requires higher doses and can result in increased side-effects. The development of dyskinesia, dopamine dysregulation syndrome, and impulse control disorders are likely influenced by genetic variation. Identifying these variants and subsequently those individuals at risk will allow the design of tailored drug treatment plans for the individual patient.

The advances in genetics of PD over the last 15 years have been remarkable; however, as we enter this new era of genomic medicine, the applications of next-generation sequencing approaches including whole-genome sequencing will accelerate gene discovery and provide a better understanding of genetic variation in disease. We witnessed the first gene identified by exome sequencing (exome signifying the $1 \%$ of the genome which encodes proteins) in 2011, with two independent groups nominating a mutation in the VPS35 gene as a cause of autosomal dominantly inherited PD $[52 \bullet, 53 \bullet]$. These studies have demonstrated the potential of genetic sequencing, and as the cost of these approaches is reduced, an ever increasing number of individuals will arrive at the clinician's door with their personal genome in hand [54].

As we stand at the epoch of individualized medicine driven by genomic profiling, we will bear witness to a transformation in patient care (Table 3). Identifying the specific variants that are driving risk of disease, whether through family- or population-based approaches, will act as determinants of diagnosis and treatment [54]. The complex etiology of PD susceptibility, involving multiple risk and protective genetic factors acting in unison, will likely reflect the therapeutic approach necessary to intervene. For example, those individuals harboring a $S N C A$-driven risk may need a knockdown therapy, those with LRRK2 may need a kinase inhibitor, and those with both may need a combination of the two. Most likely, these therapeutics with specific targets will form the underlying basis of 
Table 3 Applications of clinical genetics

The application of clinical genetics

Diagnosis

Prevention

Mechanism of disease

Model development

Target Therapeutic

Inform clinical trials

Pharmacogenomics

Table displaying the applications of genetic variation in the creation of individualized approaches for patient care and drug development, testing and application

personalized medicine and the genetic findings in PD will help direct these approaches. However, before these drugs can be developed, we need a clearer resolution of the underlying functional pathomechanisms of the disease at the cellular level to ensure the correct drug targets and reduce side-effects.

Acknowledgments I would like to thank all those who have contributed to my research, particularly the patients and families who donated DNA samples for our studies. OAR is supported by the Michael J. Fox Foundation, NINDS R01\# NS078086. The Mayo Clinic Jacksonville is a Morris K. Udall Parkinson's Disease Research Center of Excellence (NINDS P50 \#NS072187).

Disclosure The author reported no potential conflicts of interest relevant to this article.

\section{References}

Papers of particular interest, published recently, have been highlighted as:

- Of importance

1. Parkinson J. An essay on the shaking palsy. 1817. J Neuropsychiatry Clin Neurosci. 2002;14:223-36.

2. Ross OA, Farrer MJ. Pathophysiology, pleiotrophy and paradigm shifts: genetic lessons from Parkinson's disease. Biochem Soc Trans. 2005;33:586-90.

3. Lees AJ, Hardy J, Revesz T. Parkinson's disease. Lancet. 2009;373:2055-66.

4. Spillantini MG, Schmidt ML, Lee VM, Trojanowski JQ, Jakes R, Goedert M. Alpha-synuclein in Lewy bodies. Nature. 1997;388: 839-40.

5. Rascol O, Lozano A, Stern M, Poewe W. Milestones in Parkinson's disease therapeutics. Mov Disord. 2011;26:1072-82.

6. Hughes AJ, Daniel SE, Kilford L, Lees AJ. Accuracy of clinical diagnosis of idiopathic Parkinson's disease: a clinico-pathological study of 100 cases. J Neurol Neurosurg Psychiatry. 1992;55: $181-4$.

7. Lees AJ. The relevance of the Lewy body to the pathogenesis of idiopathic Parkinson's disease: accuracy of clinical diagnosis of idiopathic Parkinson's disease. J Neurol Neurosurg Psychiatry. 2012;83:954-5.
8. Gelb DJ, Oliver E, Gilman S. Diagnostic criteria for Parkinson disease. Arch Neurol. 1999;56:33-9.

9. Dickson DW, Braak H, Duda JE, et al. Neuropathological assessment of Parkinson's disease: refining the diagnostic criteria. Lancet Neurol. 2009;8:1150-7.

10. Brooks DJ. Parkinson's disease: diagnosis. Parkinsonism Relat Disord. 2012;18(Suppl 1):S31-3.

11. - Bateman RJ, Xiong C, Benzinger TL, et al. Clinical and biomarker changes in dominantly inherited Alzheimer's disease. N Engl J Med. 2012;367:795-804. This article demonstrates the neuropathology associated with the phenotype of Alzheimer's disease may occurs decades before the clinical presentation.

12. Bernheimer $\mathrm{H}$, Birkmayer $\mathrm{W}$, Hornykiewicz $\mathrm{O}$, Jellinger $\mathrm{K}$, Seitelberger F. Brain dopamine and the syndromes of Parkinson and Huntington. Clinical, morphological and neurochemical correlations. J Neurol Sci. 1973;20:415-55.

13. Langston JW. The Parkinson's complex: Parkinsonism is just the tip of the iceberg. Ann Neurol. 2006;59:591-6.

14. Gosal D, Ross OA, Toft M. Parkinson's disease: the genetics of a heterogeneous disorder. Eur J Neurol. 2006;13:616-27.

15. Polymeropoulos $\mathrm{MH}$, Lavedan C, Leroy E, et al. Mutation in the alpha-synuclein gene identified in families with Parkinson's disease. Science. 1997;276:2045-7.

16. Ross OA, Braithwaite AT, Skipper LM, et al. Genomic investigation of alpha-synuclein multiplication and parkinsonism. Ann Neurol. 2008;63:743-50.

17. Singleton AB, Farrer M, Johnson J, et al. Alpha-synuclein locus triplication causes Parkinson's disease. Science. 2003;302:841.

18. Maraganore DM, de Andrade M, Elbaz A, et al. Collaborative analysis of alpha-synuclein gene promoter variability and Parkinson disease. JAMA. 2006;296:661-70.

19. Ross OA, Gosal D, Stone JT, et al. Familial genes in sporadic disease: common variants of alpha-synuclein gene associate with Parkinson's disease. Mech Ageing Dev. 2007;128:378-82.

20. • Rhinn H, Qiang L, Yamashita T, et al. Alternative alpha-synuclein transcript usage as a convergent mechanism in Parkinson's disease pathology. Nat Commun. 1084;2012:3. Rhinn and colleagues propose a long 3UTR transcript as the toxic SNCA species in $P D$.

21. Lewis J, Melrose H, Bumcrot D, et al. In vivo silencing of alphasynuclein using naked siRNA. Mol Neurodegener. 2008;3:19.

22. Bonifati V. Autosomal recessive parkinsonism. Parkinsonism Relat Disord. 2012;18(Suppl 1):S4-6.

23. Farrer MJ. Genetics of Parkinson disease: paradigm shifts and future prospects. Nat Rev Genet. 2006;7:306-18.

24. - Geisler S, Holmstrom KM, Skujat D, et al. PINK1/Parkinmediated mitophagy is dependent on VDAC1 and p62/SQSTM1. Nat Cell Biol. 2010;12:119-31. This paper places PINK1 and Parkin in a common pathway related to labeling and degradation of damaged mitochondria (mitophagy).

25. Kawajiri S, Saiki S, Sato S, et al. PINK1 is recruited to mitochondria with parkin and associates with LC3 in mitophagy. FEBS Lett. 2010;584:1073-9.

26. Narendra DP, Jin SM, Tanaka A, et al. PINK1 is selectively stabilized on impaired mitochondria to activate Parkin. PLoS Biol. 2010;8:e1000298.

27. Jin SM, Youle RJ. PINK1- and Parkin-mediated mitophagy at a glance. J Cell Sci. 2012;125:795-9.

28. Paisan-Ruiz C, Jain S, Evans EW, et al. Cloning of the gene containing mutations that cause PARK8-linked Parkinson's disease. Neuron. 2004;44:595-600.

29. Zimprich A, Biskup S, Leitner P, et al. Mutations in LRRK2 cause autosomal-dominant parkinsonism with pleomorphic pathology. Neuron. 2004;44:601-7.

30. Ross OA, Toft M, Whittle AJ, et al. Lrrk2 and Lewy body disease. Ann Neurol. 2006;59:388-93. 
31. Di Fonzo A, Rohe CF, Ferreira J, et al. A frequent $L R R K 2$ gene mutation associated with autosomal dominant Parkinson's disease. Lancet. 2005;365:412-5.

32. Gilks WP, Abou-Sleiman PM, Gandhi S, et al. A common LRRK2 mutation in idiopathic Parkinson's disease. Lancet. 2005;365: 415-6.

33. Nichols WC, Pankratz N, Hernandez D, et al. Genetic screening for a single common LRRK2 mutation in familial Parkinson's disease. Lancet. 2005;365:410-2.

34. Kachergus J, Mata IF, Hulihan M, et al. Identification of a novel LRRK2 mutation linked to autosomal dominant parkinsonism: evidence of a common founder across European populations. Am J Hum Genet. 2005;76:672-80.

35. Lesage S, Durr A, Tazir M, et al. LRRK2 G2019S as a cause of Parkinson's disease in North African Arabs. N Engl J Med. 2006; 354:422-3.

36. Ozelius LJ, Senthil G, Saunders-Pullman R, et al. LRRK2 G2019S as a cause of Parkinson's disease in Ashkenazi Jews. N Engl J Med. 2006;354:424-5.

37. Toft M, Mata IF, Kachergus JM, Ross OA, Farrer MJ. LRRK2 mutations and Parkinsonism. Lancet. 2005;365:1229-30.

38. West AB, Moore DJ, Biskup S, et al. Parkinson's disease-associated mutations in leucine-rich repeat kinase 2 augment kinase activity. Proc Natl Acad Sci USA. 2005;102:16842-7.

39. Di Fonzo A, Wu-Chou YH, Lu CS, et al. A common missense variant in the LRRK2 gene, Gly2385Arg, associated with Parkinson's disease risk in Taiwan. Neurogenetics. 2006;7:133-8.

40. Ross OA, Wu YR, Lee MC, et al. Analysis of Lrrk2 R1628P as a risk factor for Parkinson's disease. Ann Neurol. 2008;64:88-92.

41. - Ross OA, Soto-Ortolaza AI, Heckman MG, et al. Association of LRRK2 exonic variants with susceptibility to Parkinson's disease: a case-control study. Lancet Neurol. 2011;10:898-908. This large GEO-PD consortium effort identifies common risk and protective haplotypes in LRRK2.

42. Healy DG, Falchi M, O'Sullivan SS, et al. Phenotype, genotype, and worldwide genetic penetrance of LRRK2-associated Parkinson's disease: a case-control study. Lancet Neurol. 2008;7:583-90.

43. Hulihan MM, Ishihara-Paul L, Kachergus J, et al. LRRK2 Gly2019Ser penetrance in Arab-Berber patients from Tunisia: a case-control genetic study. Lancet Neurol. 2008;7:591-4.

44. Carmine Belin A, Westerlund M, Sydow O, et al. Leucine-rich repeat kinase 2 (LRRK2) mutations in a Swedish Parkinson cohort and a healthy nonagenarian. Mov Disord. 2006;21:1731-4.

45. Nishioka K, Ross OA, Ishii $\mathrm{K}$, et al. Expanding the clinical phenotype of SNCA duplication carriers. Mov Disord. 2009;24: 1811-9.

46. - Nalls MA, Plagnol V, Hernandez DG, et al. Imputation of sequence variants for identification of genetic risks for Parkinson's disease: a meta-analysis of genome-wide association studies. Lancet. 2011;377:641-9. This large meta-analysis of PD GWA studies nominates eleven risk loci for sporadic PD.

47. Farrer M, Maraganore DM, Lockhart P, et al. Alpha-synuclein gene haplotypes are associated with Parkinson's disease. Hum Mol Genet. 2001;10:1847-51.
48. Skipper L, Wilkes K, Toft M, et al. Linkage disequilibrium and association of MAPT H1 in Parkinson disease. Am J Hum Genet. 2004;75:669-77.

49. Wider C, Vilarino-Guell C, Jasinska-Myga B, et al. Association of the MAPT locus with Parkinson's disease. Eur J Neurol. 2010;17:483-6.

50. - Elbaz A, Ross OA, Ioannidis JP, et al. Independent and joint effects of the MAPT and SNCA genes in Parkinson disease. Ann Neurol. 2011;69:778-92. This GEO-PD consortium effort demonstrates that the common risk alleles at the SNCA and MAPT loci do not interact.

51. - Lill CM, Roehr JT, McQueen MB, et al. Comprehensive research synopsis and systematic meta-analyses in Parkinson's disease genetics: the PDGene database. PLoS Genet. 2012;8:e1002548. Lill and colleagues analyze over 800 independent studies as meta-analysis on the PDgene website.

52. - Vilarino-Guell C, Wider C, Ross OA, et al. VPS35 mutations in Parkinson disease. Am J Hum Genet. 2011;89:162-7. The first report of a novel PD gene using Next-Generation sequencing approaches.

53. - Zimprich A, Benet-Pages A, Struhal W, et al. A mutation in VPS35, encoding a subunit of the retromer complex, causes lateonset Parkinson disease. Am J Hum Genet. 2011;89:168-75. The first report of a novel PD gene using Next-Generation sequencing approaches.

54. Ross OA, Farrer MJ. Parkinson disease: Parkinson diseasemoving beyond association. Nat Rev Neurol. 2010;6:305-7.

55. Pankratz N, Wilk JB, Latourelle JC, et al. Genomewide association study for susceptibility genes contributing to familial Parkinson disease. Hum Genet. 2009;124:593-605.

56. Hamza TH, Zabetian CP, Tenesa $\mathrm{A}$, et al. Common genetic variation in the HLA region is associated with late-onset sporadic Parkinson's disease. Nat Genet. 2010;42:781-5.

57. Satake W, Nakabayashi Y, Mizuta I, et al. Genome-wide association study identifies common variants at four loci as genetic risk factors for Parkinson's disease. Nat Genet. 2009;41:1303-7.

58. Saad M, Lesage S, Saint-Pierre A, et al. Genome-wide association study confirms BST1 and suggests a locus on 12q24 as the risk loci for Parkinson's disease in the European population. Hum Mol Genet. 2011;20:615-27.

59. Do CB, Tung JY, Dorfman E, et al. Web-based Genome-wide association study identifies two novel loci and a substantial genetic component for Parkinson's disease. PLoS Genet. 2011;7: e1002141.

60. - Plagnol V, Nalls MA, Bras J, et al. A two-stage meta-analysis identifies several new loci for Parkinson's disease. PLoS Genet. 2011;7:e1002142. This follow-up to the large meta-analysis of $P D$ GWA studies nominates risk loci for sporadic PD.

61. - Pankratz N, Beecham GW, DeStefano AL, et al. Meta-analysis of Parkinson's disease: identification of a novel locus, RIT2. Ann Neurol. 2012;7:370-84. This large meta-analysis of PD GWA studies nominates RIT2 as a risk loci for sporadic PD. 